\title{
Hybridization of Guided Surface Acoustic Modes in Unconsolidated Granular Media by a Resonant Metasurface
}

\author{
Antonio Palermo, ${ }^{1,5}$ Sebastian Krödel, ${ }^{2}$ Kathryn H. Matlack, ${ }^{2,3}$ Rachele Zaccherini, ${ }^{4}$ Vasilis K. Dertimanis, ${ }^{4}$ \\ Eleni N. Chatzi, ${ }^{4}$ Alessandro Marzani, ${ }^{1, *}$ and Chiara Daraio ${ }^{5, \dagger}$ \\ ${ }^{1}$ Department of Civil, Chemical, Environmental and Materials Engineering-DICAM, \\ University of Bologna, Bologna 40136, Italy \\ ${ }^{2}$ Department of Mechanical and Process Engineering, ETH Zürich, Zürich 8092, Switzerland \\ ${ }^{3}$ Department of Mechanical Science and Engineering, University of Illinois at Urbana-Champaign, \\ Urbana, Illinois 61801, USA \\ ${ }^{4}$ Department of Civil, Environmental and Geomatic Engineering, ETH Zürich, Zürich 8093, Switzerland \\ ${ }^{5}$ California Institute of Technology, Division of Engineering and Applied Science, \\ Pasadena, California 91125, USA
}

(Received 3 November 2017; revised manuscript received 1 April 2018; published 17 May 2018)

\begin{abstract}
We investigate the interaction of guided surface acoustic modes (GSAMs) in unconsolidated granular media with a metasurface, consisting of an array of vertical oscillators. We experimentally observe the hybridization of the lowest-order GSAM at the metasurface resonance, and note the absence of mode delocalization found in homogeneous media. Our numerical studies reveal how the stiffness gradient induced by gravity in granular media causes a down-conversion of all the higher-order GSAMs, which preserves the acoustic energy confinement. We anticipate these findings to have implications in the design of seismic-wave protection devices in stratified soils.
\end{abstract}

DOI: 10.1103/PhysRevApplied.9.054026

\section{INTRODUCTION}

Acoustic waves confined at the free surface of an elastic medium are observed in multiple natural phenomena, such as seismic surface waves released by an earthquake [1] or ocean waves in deep water [2]. Surface acoustic waves (SAWs) are also exploited in different technological applications, for nondestructive testing of materials and structures [3], as well as for the realization of delay lines, filters, transducers, and converters in electronic components [4,5]. In homogenous media, the confinement mechanism of sagittal polarized SAWs depends on the presence of a stress-free interface, the surface, where bulk shear and longitudinal modes couple to generate the elliptical particle motion of Rayleigh waves [1]. When the free-stress surface condition is perturbed by the introduction of an array of local resonators, also referred as "metasurface" [6-9], the wave confinement mechanism can be controlled. Highly localized modes arise due to the coupling of Rayleigh waves with the vertical oscillators at resonance. Conversely, the complete SAWs' delocalization is observed in a narrow frequency region above the resonance, as a result of a classic "avoided crossing" behavior between Rayleigh-like

\footnotetext{
*Corresponding author. alessandro.marzani@unibo.it

Corresponding author. daraio@ caltech.edu
}

solutions with opposite phases [10]. Within this frequency range, Rayleigh waves are deflected away from the surface, in the form of shear vertically polarized waves. The Rayleigh-to-shear wave conversion has been predicted and observed at microscales [10] and macroscales [11], and its use has been proposed for applications in sensing [12] and seismic waves deflection [13,14].

However, most natural and human-made materials are not homogeneous. For these materials, localization can be induced not only by the presence of a mechanically free surface, but also by the inhomogeneous elastic properties. In unconsolidated granular media under gravity load, the shear $G$ and the bulk $B$ elastic moduli profiles exhibit a power-law dependence on the compacting pressure $p=\rho g z$, i.e., $G, B \propto p^{\alpha}$, where $\rho$ is the medium density, $g$ the gravitational constant, and $z$ the considered depth. This peculiar vertical stiffness profile induces a further localization mechanism, which consists of the upward bending of the acoustic waves towards the surface, commonly referred to as the "mirage" effect [15]. As a result, elliptical sagittal polarized waves are guided between the free surface and the increasingly stiffer material. Experimental observations of such guided surface acoustic modes (GSAMs) in granular media have shown uncommon low propagation velocities (approximately $50 \mathrm{~m} / \mathrm{s}$ ), ascribed to the soft near-surface layers confined at low pressures close to the medium jamming transition [16]. The same observations have been used to estimate the elastic 
power-law coefficients, which significantly deviate from the classical prediction given from the Hertz contact theory. The fitted coefficients have been employed in ad hoc theoretical frameworks, to analytically predict the GSAM dispersive properties $[16,17]$ and in numerical simulations, to replicate small-scale laboratory models for seismic-wave propagation in granular media [18].

Despite the extensive investigation on the dynamics of unconsolidated granular media [19], the interaction of GSAMs with local resonances remains unexplored. In this work, we aim at understanding (i) how GSAMs hybridize with local resonances, and (ii) if a resonant metasurface can induce energy delocalization, like in homogeneous media $[10,13,14]$.

\section{RESULTS}

\section{A. Experimental investigation and dispersion analysis}

We design an experimental setup to investigate sagittal polarized guided surface acoustic modes (GSAMs) interacting with surface resonances. The setup consists of a wooden box of dimensions $2000 \times 1500 \times 1000 \mathrm{~mm}$, filled with a granular medium of silica microparticles, up to $830 \mathrm{~mm}$ in height [see Fig. 1(a), and Supplemental Material [20] for further details]. The microparticles are sieved glass beads with a controlled diameter of $150 \mu \mathrm{m}$ and density of $1600 \mathrm{~kg} / \mathrm{m}^{3}$. Similar setups have been used to analyze the propagation of GSAMs $[18,21]$ and to probe the jamming transition of granular media [16]. We embed subwavelength resonators [14] (height $24 \mathrm{~mm}$, diameter $20 \mathrm{~mm}$ ) below the surface of the granular medium. The resonators consist of a rigid cylindrical shell and a soft spring, both 3D printed in acrylonitrile butadiene styrene, and a steel cylindrical mass [inset, Fig. 1(a)]. The steel cylinder (height $12 \mathrm{~mm}$, diameter $12 \mathrm{~mm}$ ) is inserted in the outer shell after the 3D printing process. The soft spring is designed with a trusslike geometry, which allows tuning the first resonator vertical frequency by changing the length and angle of the truss elements [see Fig. S2(a) in the Supplemental Material [20]]. Higher-order vertical and flexural resonant modes are neglected in the resonator design process. We produce 100 resonators and arrange them on the granular medium surface in a $20 \times 5$ rectangular grid with a spacing of $24 \mathrm{~mm}$ in the $x$ - and $y$-directions [see Fig. 1(a)]. The resonators embedded in the granular medium have a measured first vertical resonance frequency $f_{r}=410 \mathrm{~Hz}$ [see Fig. S2(b) in Supplemental Material [20]]. We remark that the GSAM-resonator coupling is independent from the particular choice of the lattice arrangement, i.e., a rectangular grid, as long as the subwavelength regime of the resonators is ensured.

An 8-mm-diameter metallic rod is attached to an electromagnetic shaker and buried in the granular medium to excite the GSAMs. The shaker is driven by a waveform
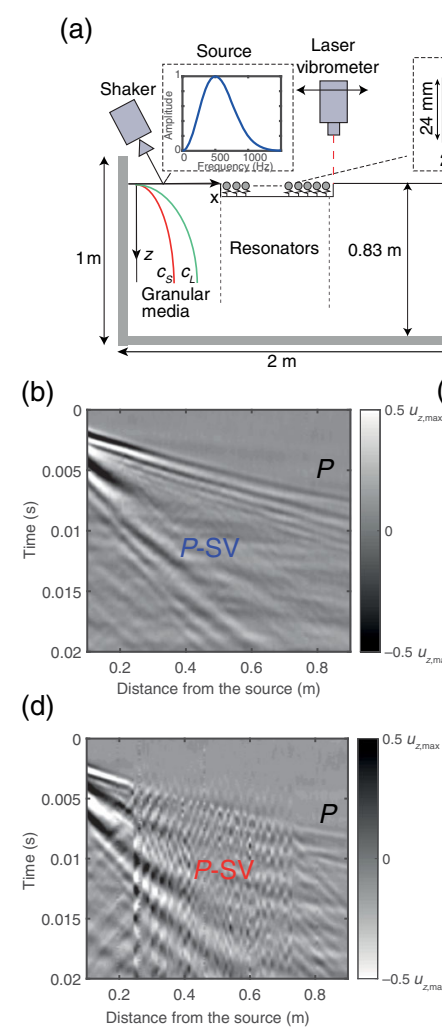

(c)
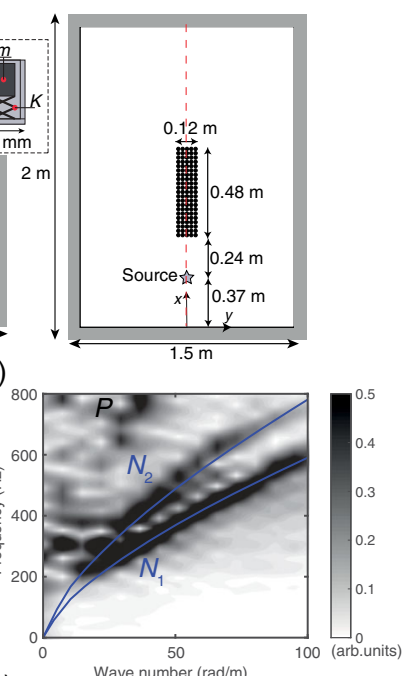

(e)

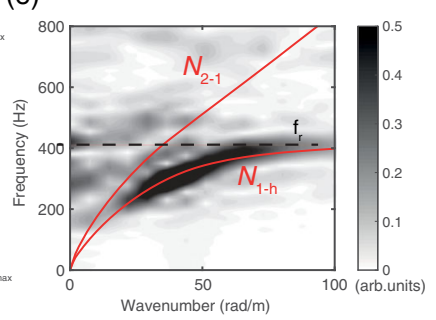

FIG. 1. Experimental characterization of GSAMs interacting with surface resonances. (a) Schematic of the experimental setup. (b) Seismograph of a Ricker pulse propagating in the granular medium. (c) Dispersion curves of low-order GSAMs traveling in the granular medium: the gray color map is the 2D Fouriertransform magnitude of the experimental seismograph, while the blue curves are the lowest-order modes $\left(N_{1}, N_{2}\right)$ calculated using the Bloch-FE numerical model. (d) Seismograph of a Ricker pulse propagating through the surface resonances embedded in the granular medium. (e) Dispersion curves of low-order GSAMs interacting with surface resonances: the gray color map is the 2D Fourier-transform magnitude of the experimental seismograph, while the red curves are the lowest-order modes $\left(N_{1-h}, N_{2-1}\right)$ calculated using the numerical model. Color maps are scaled with respect to their maximum value.

generator, which produces a Ricker waveform centered at $500 \mathrm{~Hz}$. We measure the surface velocity field generated by the point source using a laser-Doppler vibrometer mounted on a scanning stage.

We perform point measurements on the surface of the granular medium with and without resonators, and record the wave field across the symmetry axis of the box at constant steps $\Delta x=6.6 \mathrm{~mm}$, starting $100 \mathrm{~mm}$ away from the source for an overall length of $850 \mathrm{~mm}$. All measurements are carried out with small-amplitude excitations to generate linear GSAMs, as in Ref. [16]. For the free-surface conditions (without resonators), two coherent and distinct wave trains are recognized [Fig. 1(b)]: the first is a highfrequency (approximately 700-800 Hz) quasicompressional wave $(P)$ traveling at approximately $150 \mathrm{~m} / \mathrm{s}$ (see 
Supplemental Material [20]); the second event is a packet of sagittal compressional-shear vertical $(P-\mathrm{SV})$ waves, spread in the frequency range between $200-650 \mathrm{~Hz}$ with low phase velocities between $35-90 \mathrm{~m} / \mathrm{s}$.

We restrict our analysis to the dynamics of these $P$-SV waves, which can be described using the GSAM theory developed in Ref. [17]. In the GSAM framework, the granular medium is modeled as a continuous medium. The bulk shear $c_{S}(z)$ and longitudinal $c_{S}(z)$ speed profiles are modeled with a power-law dependency on the depth $c_{S, L}(z)=\gamma_{S, L}(\rho g z)^{\alpha_{S, L}}$. In this medium, the governing equations for the displacement $U=\left\{u_{x}(z), u_{z}(z)\right\} e^{i(\omega t-k x)}$ in the sagittal plane are

$$
\begin{aligned}
& {\left[c_{S}^{2}(z) u_{x}^{\prime}\right]^{\prime}+\left[(\omega / k)^{2}-c_{L}^{2}(z)\right] u_{x}} \\
& \quad=i\left\{c_{L}^{2}(z)-2 c_{S}^{2}(z) u_{z}^{\prime}+\left[c_{S}^{2}(z) u_{z}\right]^{\prime}\right\}, \\
& {\left[c_{L}^{2}(z) u_{z}^{\prime}\right]^{\prime}+\left[(\omega / k)^{2}-c_{S}^{2}(z)\right] u_{z}} \\
& \quad=i\left(c_{S}^{2}(z) u_{x}^{\prime}-\left\{\left[c_{L}^{2}(z)-2 c_{S}^{2}(z)\right] u_{z}\right\}^{\prime}\right),
\end{aligned}
$$

where $\omega$ denotes the wave angular frequency, $k$ the wave number along the direction of wave propagation, $i$ the imaginary unit, and the prime indicates derivative with respect to the $z$ coordinate. The GSAM characteristic equation is analytically obtained by combining the governing Eqs. (1) with the free-stress boundary conditions $\left[\sigma_{z z}(0)=0\right.$, $\left.\sigma_{x z}(0)=0\right]$ and the localization condition $U(z \rightarrow \infty)=0$.

In contrast to Ref. [17], we numerically implement and solve the characteristic equation by modeling a portion of the granular medium with a Bloch wave-finite element (FE) approach. We consider a 2D FE model of the unit cell, developed in COMSOL Multiphysics ${ }^{\circledR}$, and confine our analysis to vertically polarized surface waves by modeling a vertical stripe of the granular medium in plane-strain conditions. Within the FE framework, the Bloch form wave solution is assumed by imposing periodic Bloch-Floquet boundary conditions (BCs) on the unit cell waveguide, along the direction of wave propagation. The granular medium is modeled as a linear elastic continuum, assuming the powerlaw stiffness profile $c_{S, L}(z)=\gamma_{S, L}(\rho g z)^{\alpha_{S, L}}$ reported in Ref. [17]. Since the analyzed medium is homogenous along the wave-propagation direction, we can arbitrarily set the width $d$ of the unit cell to map the dispersive properties in the desired wave-number range $k=$ $[0-(\pi / d)]$. We set $d=0.012 \mathrm{~m}$ to span a wave-number range up to $k_{\max }=260 \mathrm{rad} / \mathrm{m}$. The unit cell has a height $h=3 \mathrm{~m}$ with perfectly matched layers (PMLs) at the bottom region to avoid wave reflection and clamped BCs at the bottom edge [see Fig. 2(a)]. Such dimensions ensure the correct representation of a semi-infinite space domain (and the related localization condition $U(z \rightarrow \infty)=0)$ in the frequency range of interest and avoid the occurrence of platelike flexural modes.

The dispersion curves for the first two slowest $P$-SV waves, $N_{1}$ and $N_{2}$, calculated using our numerical approach agree well the experimental dispersion curves obtained

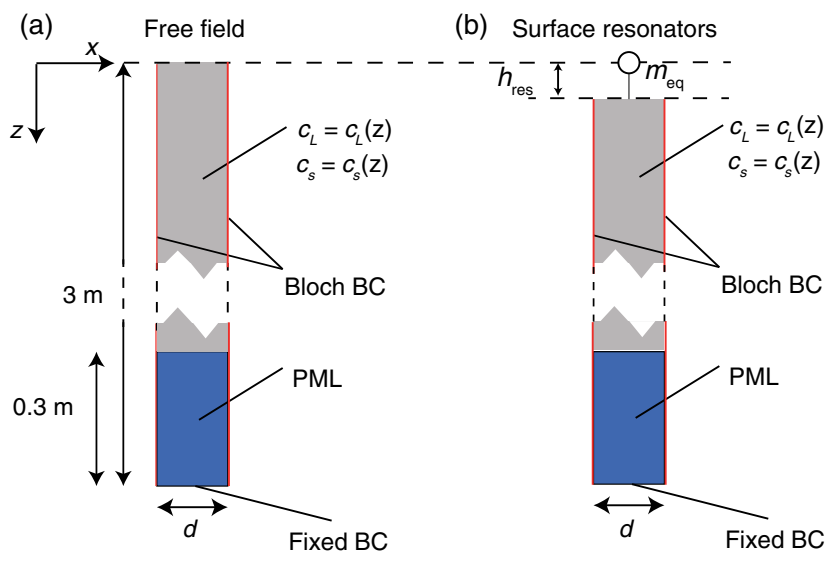

FIG. 2. Schematic of the unit cell models for the Bloch-FE approach. (a) Unit cell model for the granular medium and (b) for the granular medium with surface resonators $(d=0.012 \mathrm{~m}$ to span a wave-number range up to $k_{\max }=260 \mathrm{rad} / \mathrm{m}$ ).

from a 2D Fourier transform of the seismograph data [see Fig. 1(c)]. For the calculation of the dispersion curves, we assumed the power-law parameters $\gamma_{S}=6.42, \gamma_{L}=14.4$, $\alpha_{S}=0.31, \alpha_{L}=0.31$. The parameters are approximated from the experimental estimates given in Ref. [16] (i.e., $\gamma_{S}^{\exp }=6.42 \pm 0.13, \quad \gamma_{L}^{\exp }=14.4 \pm 0.4, \quad \alpha_{S}^{\exp }=0.316 \pm 0.006$, $\left.\alpha_{L}^{\exp }=0.31 \pm 0.01\right)$ for the same material. Here, by assuming $\alpha_{S}=\alpha_{L}$ we avoid the introduction of a characteristic spatial scale in the system [16].

The GSAM framework [17] predicts an infinite number of confined surface modes, whose order $N_{i}$ coincides with the number of zeros in their vertical displacement profile along the depth. As a result, the mode order corresponds also to the number of "phases" in the displacement depth profile. In other words, for the first mode $\left(N_{1}\right)$, the particle displacements are in phase at all depths; conversely, the depth displacement profile presents two alternating phases for the second mode $\left(N_{2}\right)$, three phases for the third mode $\left(N_{3}\right)$, and so forth [17].

However, among the plethora of modes existing in an unconsolidated medium, only the lowest-order ones can strongly couple to the resonators due to their significant vertical displacement component at the surface. Among these, the first-order mode $\left(N_{1}\right)$ converges to a Rayleigh wave solution when the inhomogeneous velocity profile accounts for a significant granular adhesion [17].

The seismograph obtained when resonators are embedded near the surface [Fig. 1(d)] demonstrates the resonance excitation induced by the train of $P$-SV waves, which leads to a clear flat branch that asymptotically approaches the resonator's frequency $f_{r}$ in the 2D Fourier-transformed domain [Fig. 1(e)]. This mode is a result of the hybridization between the first-order $P$-SV waves and the resonators, which oscillate in phase with the medium and progressively confine the energy to the surface. We remark that at the resonance frequency $f_{r}$, the 
smallest wavelength of $P$-SV waves is at least 5 times larger than the resonator spacing, thus excluding any relation with Bragg-scattering effects.

To predict the dispersive properties of this hybrid mode, we directly couple the GSAM governing equation [Eq. (1)] with the resonator dynamics, using the Bloch-wave-FE approach. The equation of motion for a resonator of mass $m$ and stiffness $K_{v}$, buried near the surface at a depth $h_{\text {res }}$ can be written as $m\left(\ddot{Z}+\ddot{u}_{z, h_{\text {res }}}\right)+K_{v} Z=0$, where $u_{z, h_{\text {res }}}$ is the displacement of the granular medium at the depth $h_{\text {res }}, Z$ is the vertical displacement of the oscillator mass relative to the granular medium, and the overdot indicates the time derivative. Each resonator exerts a dynamic stress on the granular medium $\sigma_{z z, h_{\mathrm{res}}}=\left(K_{v} / A\right)\left[\omega^{2} /\left(\omega_{r}^{2}-\omega^{2}\right)\right] u_{z, h_{\mathrm{res}}}$, $\sigma_{x z, h_{\text {res }}}=0$, with $\omega_{r}=2 \pi f_{r}$ and $A$ the surface area over which the resonator stress is considered distributed uniformly $\left(24 \times 24 \mathrm{~mm}^{2}\right.$ in this case). Such dynamic stress can be seen as a boundary condition at surface depth $h_{\text {res }}$. We remark that similar resonant surface conditions have been successfully used to describe the hybridization of Rayleigh waves with resonances at a different length scale $[10,14]$. Additionally, at the depth $h_{\text {res }}$, the unconsolidated medium is subjected to a pressure $p_{h_{\text {res }}}=\rho g h_{\text {res }}$, which acts as an adhesive force between the grains, ensuring nonzero elastic properties for the granular medium. Within the FE framework, the resonator dynamic is modeled by means of a trussmass point element [see Fig. S2(b) in Supplemental Material [20] ], of mass $m_{\mathrm{eq}}=(m / A) d$ (per unitary thickness) and resonance frequency $f_{r}$. The truss-mass resonator exerts a uniform dynamic vertical stress $\sigma_{z z, h_{\text {res }}}$ over the length $d$ equivalent to the uniform stress of a 3D resonator of mass $m$ acting on its reference area $A$ [see Fig. 2(b)].

The hybridized $\left(N_{1-h}\right)$ mode, calculated using our numerical model, is in excellent agreement with the experimental data [Fig. 1(e)]. We observe that the dispersion of this confined mode is akin to the lower branch of Rayleigh waves hybridized by surface resonances [10,14]. For Rayleigh waves, the lower branch is accompanied by a repelling upper branch, which leads to an avoided crossing behavior and a related surface-to-shear wave-conversion phenomenon above the resonance frequency.

In our experiments, higher-frequency $P$-SV modes are weakly excited and most of the high-frequency energy is transported in the form of quasicompressional $(P)$ waves. Moreover, large surface velocities measured at the resonance frequency saturate the experimental $f-k$ spectrum, rendering the investigation of higher-frequency solutions difficult. To gain insight into the propagation of higherfrequency GSAMs and into the possible wave-conversion phenomena around the resonance frequency, we perform FE time-transient simulations.

\section{B. Time-transient numerical simulations}

Direct simulation of the experimental setup agrees with the measurement data (see Supplemental Material [20]).

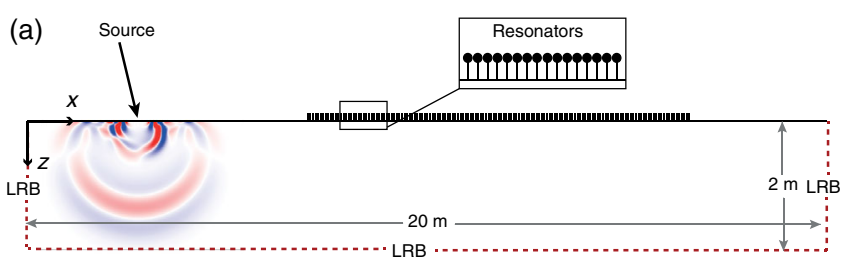

(b)

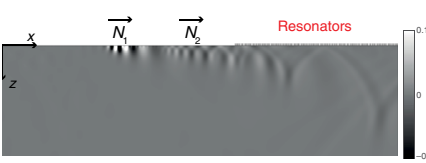

(c)

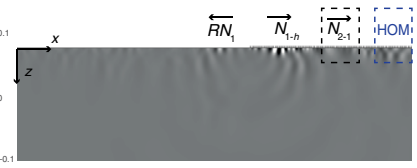

FIG. 3. Transient simulations of a Ricker pulse traveling in a granular medium with surface resonances. (a) Schematic of the numerical model. (b) Snapshot of the vertical displacement field of low-order $\left(N_{1}, N_{2}\right)$ GSAMs traveling in the free granular medium. (c) Snapshot of the vertical displacement field of loworder GSAMs interacting with surface resonances. $R N_{1}$ indicates the reflected $N_{1}$ mode due to the impedance mismatch generated by the resonators. $N_{1-h}, N_{2-1}$ indicate the low-order GSAMs traveling within the surface resonant region, and HOM the higher-order GSAM. Color maps in Fig. 3. (b),(c) are scaled with respect to the maximum vertical displacement at the source.

However, due to the finite dimensions of the box, such simulation does not fully disclose the GSAMs highfrequency hybridization. Thus, we employ a larger 2D FE model to obtain accurate numerical predictions of such highfrequency wave-conversion phenomena. We model a 2D strip of the granular medium in plane-strain conditions, assuming the previously considered power-law elastic profiles and modeling the resonators as truss-point masses with a single longitudinal resonance [see Fig. 3(a)]. This model is based on the unit cell description used for the dispersion relation calculation. Low-reflective-boundary (LRB) conditions are employed to minimize boundary reflections. A point source driven by a Ricker pulse placed at sufficient distance from the model boundaries generates the surface waves. In accordance with the experimental evidence, the source mainly excites the lower-order GSAMs $\left(N_{1}\right.$ and $\left.N_{2}\right)$, which spatially separate due to the different wave speeds [see Fig. 3(b)]. Once the train of GSAMs reaches the resonators, part of the wave energy is reflected [mode $R N_{1}$ in Fig. 3(c)], mainly belonging to the $N_{1}$ mode. This is highlighted by the 2D Fourier transform of the surface vertical displacement wave field of the reflected signal, shown in Fig. 4(a). Indeed, the hybridization phenomenon introduces a phase shift of $\pi$ on the incident $N_{1}$ mode causing the reflection of the wave field around the resonant frequency.

Within the surface resonant region, the hybridized mode $\left(N_{1-h}\right)$, slowed down by the surface resonances, is preceded by a second mode $\left(N_{2-1}\right)$ which travels almost undisturbed [Fig. 3(c)]. The mode $N_{1-h}$ gets confined to the surface but preserves the single-zero depth profile (i.e., the single-phase profile) of a $N_{1}$ mode across the different frequency ranges. This is shown by the mode shapes 
(a)

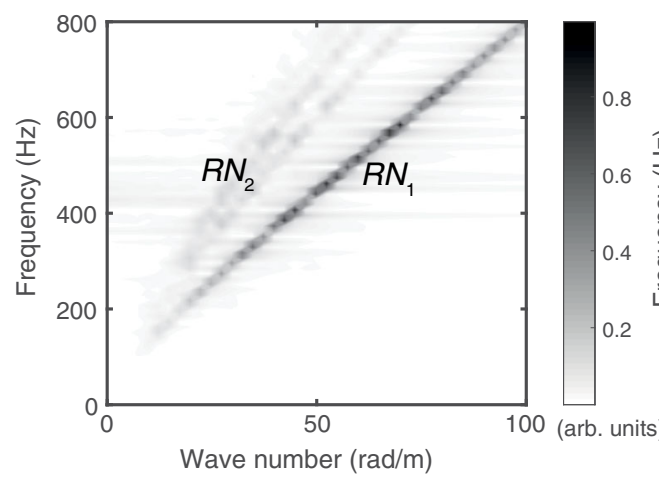

(b)

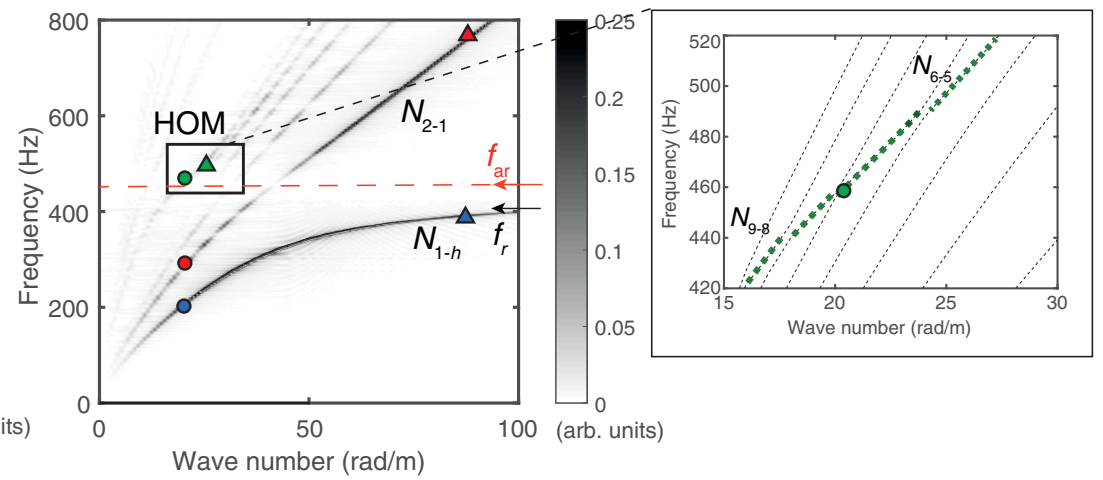

FIG. 4. Dispersion curves computed from 2D Fourier transforms of the time-domain surface displacement field. (a) Dispersion curves of the surface wave field reflected by the resonators. (b) Dispersion curves of the surface wave field within the resonant region. In the inset is shown the HOM as predicted from the Bloch-FE approach. Color maps are scaled with respect to their maximum value.

extracted from the Bloch-FE dispersion relation at different frequency points [see Fig. 5(a)]. Conversely, the mode $N_{2-1}$ presents a displacement profile that changes across the frequency spectrum. In the low frequency range, the mode profile presents two zeros (i.e., two alternating displacement phases). The position of the uppermost zero is progressively shifted towards the surface, for frequencies approaching the antiresonance $\left(f_{\mathrm{ar}}=460 \mathrm{~Hz}\right)$ [see top inset Fig. 5(a)]. At the antiresonance, the surface displacement is zero while for higher frequencies the depth profile of the $N_{2-1}$ mode no longer presents two distinct displacement phases but reduces to the single-phase profile of a first order $\left(N_{1}\right.$-like) GSAM. Indeed, the out-of-phase motion gets confined to the surface resonators, as evidenced in the bottom inset of Fig. 5(a).
In addition, we observe a clear gap in the $f-k$ spectrum at the antiresonance frequency [Fig. 4(b)]. This suggests that all the GSAMs have a vanishing surface displacement at the antiresonance. In fact, we observe an analogous change of the displacement profile across the antiresonance frequencies for all the analyzed higher-order GSAMs modes, $N_{3-2}, N_{4-3}, N_{5-4}$. All these modes present the expected null surface displacement at the antiresonance frequency [see Fig. 5(b)] with the "missing" out-of-phase motion confined in the resonators for higher frequencies [see bottom inset of Fig. 5(a)]. We concisely label this phenomenon "down-conversion" of the higher-order GSAM, since the depth profile of each $i$ th mode loses one of its phases across the antiresonance and is converted into the profile of the related $i$ th -1 mode.
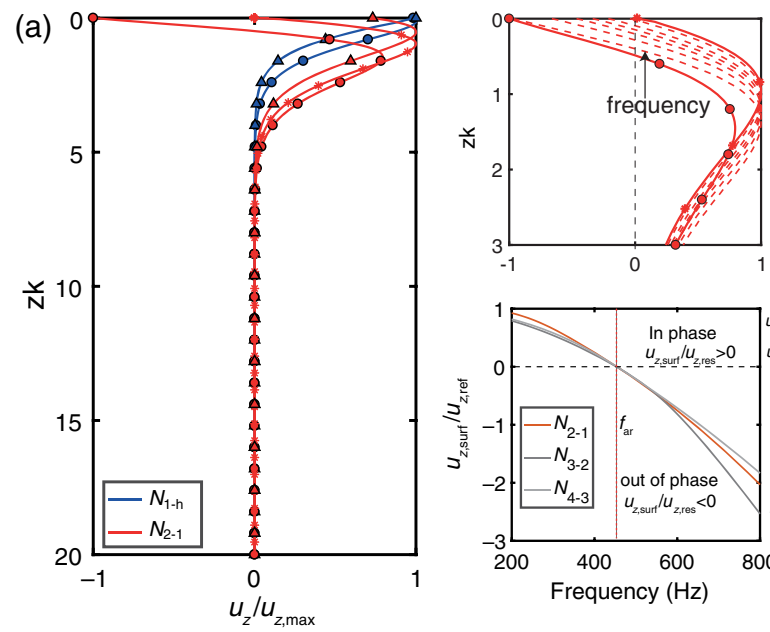

(b)
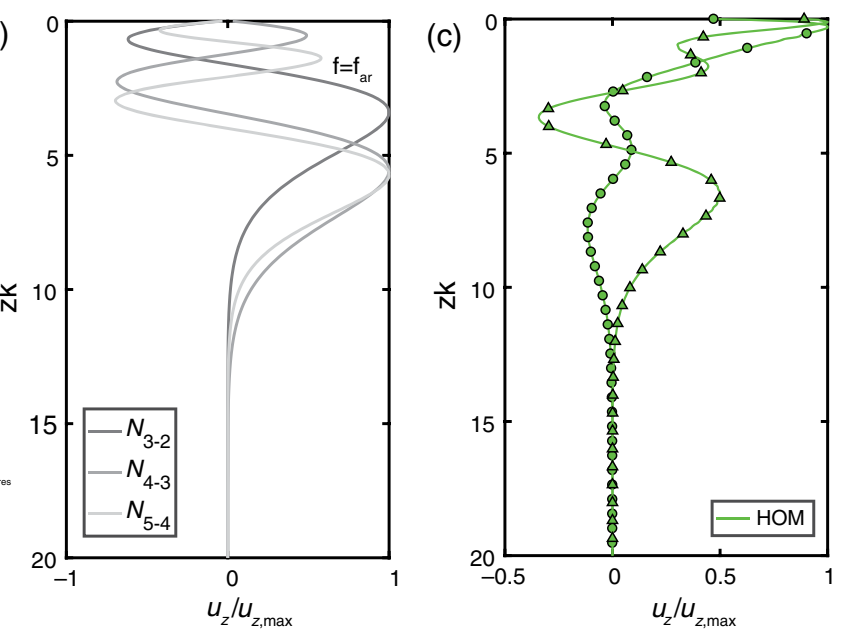

FIG. 5. Mode shapes of GSAMs interacting with surface resonances. (a) $N_{1-h}$ and $N_{2-1}$ mode shapes at the selected frequency shown in Fig. 3(b). The upper inset shows the $N_{2-1}$ shift of the displacement zero position towards the surface for increasing frequencies. The lower inset shows the ratio between the vertical displacement of the medium surface and the vertical displacement of the resonating mass for different mode shapes $\left(N_{2-1}, N_{3-2}, N_{4-3}\right)$ at different frequencies. (b) Mode shapes of $N_{3-2}, N_{4-3}, N_{5-4}$ at the antiresonance frequency. All the mode shapes present a vanishing displacement at the surface. (c) Mode shapes of the HOM mode at the frequency values shown in Fig. 4(b). 
In addition, we also observe a significant trace associated with a peculiar higher-order mode (HOM) lying between the resonance and antiresonance frequency [inset, Fig. 4(b)]. The mode stems from the progressive coupling of multiple higher-order GSAMs, specifically the $N_{9-8}$ to $N_{6-5}$, with the surface resonances [Fig. 5(b) inset]. The mode presents a depth profile maximized at the surface [Fig. 5(c)] and propagates with a group velocity significantly lower than the surrounding HOMs. Similar dispersive properties are also observed for the upper repelling branch of Rayleigh waves in homogenous media interacting with surface resonances [10]. However, in the homogenous media, the high-frequency branch gets progressively delocalized from the surface for frequencies approaching the resonance. The surface wave delocalization stems from the increasing phase velocity $c_{R h}$ of the hybridized Rayleigh modes, which progressively approaches the elastic medium shear velocity $c_{S}$. When the condition $c_{R h}=c_{S}$ is reached, the hybrid-Rayleigh waves are deflected away from the surface, in the form of shear vertically polarized waves [10].

In the unconsolidated granular medium, the formation of shear waves leaking in the bulk is prevented by the continuous variation of the shear $c_{S}$ and longitudinal $c_{L}$ velocity profile, which in turn causes the coupling between shear and compressional waves at each point of the inhomogeneous media [17]. The absence of energy leakage is confirmed by the $f-k$ spectrum of the displacement field recovered after the resonant region, which does not show any significant energy gap above the resonant frequency [see Fig. S4(b) in the Supplemental Material [20] ].

\section{CONCLUSIONS}

In conclusion, we describe numerically and experimentally how sagittal GSAMs interact with a metasurface of vertical oscillators in an unconsolidated granular medium. Resonant boundary conditions lead to the existence of two strongly hybridized modes, which are akin to the repelling hybrid Rayleigh waves. However, the inhomogeneous profile of the granular media ensures that the wave energy remains channeled below the resonant boundary, even at the antiresonance, where the surface displacement is vanishing.

Since these results do not strictly depend on the granular nature of the medium, one can conclude that similar phenomena can be observed in any natural or artificial material with an inhomogeneous power-law elastic profile. In particular, the design of SAW devices on layered functionally graded materials can benefit from the knowledge of the dispersive properties of metasurfaces on inhomogeneous materials. In addition, since stratified soils generally present inhomogeneous profiles at the seismic scale, the design of large-scale metadevices for seismic isolation purposes $[13,14,22,23]$ should consider the effect of material inhomogeneity. Although we recognize that the power-law stiffness profile of a granular medium does not necessarily represent all inhomogeneous soils, we still remark its relevance for the seismic-scale scenario, where similar depth dependencies are commonly adopted [24,25]. In the future, large-scale experiments would be helpful to assess the effectiveness of resonant elements in isolating surface seismic waves.

\section{ACKNOWLEDGMENTS}

We acknowledge Viktor Thöni for the help in the construction of the experimental setup and for the initial measurements of the GSAMs velocities. We thank Virginia von Streng for the help in designing the 3D printed resonators and Isotta Carpi for the help in the preliminary numerical models. This work was partially supported by the Swiss National Science Foundation (Grant No. 164375) to C. D., by the ETH Research Grant (49 17-1) to R. Z., and an ETH Postdoctoral Fellowship to K. H. M.

[1] K.F. Graff, Wave Motion in Elastic Solids (Dover Publications, The Clarendon Press, Oxford, 1975).

[2] S. R. Massel, Ocean Surface Waves: Their Physics and Prediction (World Scientific Publishing, Singapore, 1996).

[3] G. Kim, C. W. In, J. Y. Kim, K. E. Kurtis, and L. J. Jacobs, Air-coupled detection of nonlinear Rayleigh surface waves in concrete-Application to microcracking detection, NDT\&E Int. 67, 64 (2014).

[4] J. D. Maines and E. G. S. Paige, Surface-acoustic-wave devices for signal processing applications, Proc. IEEE 64, 639 (1976).

[5] C. Campbell, Surface Acoustic Wave Devices and Their Signal Processing Applications (Academic Press, Boston, 1989).

[6] A. A. Maznev and V. E. Gusev, Waveguiding by a locally resonant metasurface, Phys. Rev. B 92, 115422 (2015).

[7] G. Ma, M. Yang, S. Xiao, Z. Yang, and P. Sheng, Acoustic metasurface with hybrid resonances, Nat. Mater. 13, 873 (2014).

[8] A. V. Kildishev, A. Boltasseva, and V. M. Shalaev, Planar photonics with metasurfaces, Science 339, 1232009 (2013).

[9] D. J. Colquitt, A. Colombi, R. V. Craster, P. Roux, and S. R.L. Guenneau, Seismic metasurfaces: Subwavelength resonators and Rayleigh wave interaction, J. Mech. Phys. Solids 99, 379 (2017).

[10] N. Boechler, J. K. Eliason, A. Kumar, A. A. Maznev, K. A. Nelson, and N. Fang, Interaction of a Contact Resonance of Microspheres with Surface Acoustic Waves, Phys. Rev. Lett. 111, 036103 (2013).

[11] A. Colombi, P. Roux, S. Guenneau, P. Gueguen, and R. V. Craster, Forests as a natural seismic metamaterial: Rayleigh wave bandgaps induced by local resonances, Sci. Rep. 6, 19238 (2016).

[12] A. Colombi, V. Ageeva, R. J. Smith, A. Clare, R. Patel, M. Clark, D. Colquitt, P. Roux, S. Guenneau, and R. V. Craster, Enhanced sensing and conversion of ultrasonic 
Rayleigh waves by elastic metasurfaces, Sci. Rep. 7, 6750 (2017).

[13] A. Colombi, D. Colquitt, P. Roux, S. Guenneau, and R. V. Craster, A seismic metamaterial: The resonant metawedge, Sci. Rep. 6, 27717 (2016).

[14] A. Palermo, S. Krödel, A. Marzani, and C. Daraio, Engineered metabarrier as shield from seismic surface waves, Sci. Rep. 6, 39356 (2016).

[15] C. H. Liu and S. R. Nagel, Sound in Sand, Phys. Rev. Lett. 68, 2301 (1992).

[16] X. Jacob, V. Aleshin, V. Tournat, P. Leclaire, W. Lauriks, and V.E. Gusev, Acoustic Probing of the Jamming Transition in an Unconsolidated Granular Medium, Phys. Rev. Lett. 100, 158003 (2008).

[17] V. Aleshin, V. Gusev, and V. Tournat, Acoustic modes propagating along the free surface of granular media, J. Acoust. Soc. Am. 121, 2600 (2007).

[18] L. Bodet, A. Dhemaied, R. Martin, R. Mourgues, F. Rejiba, and V. Tournat, Small-scale physical modeling of seismicwave propagation using unconsolidated granular media, Geophysics 79, T323 (2014).
[19] V. Tournat and V. E. Gusev, Acoustics of unconsolidated "Model" granular media: An Overview of recent results and several open problems, Acta Acust. Acust. 96, 208 (2010).

[20] See Supplemental Material at http://link.aps.org/ supplemental/10.1103/PhysRevApplied.9.054026 for details on the experimental setup, 3D-printed resonators design, and finite-element models.

[21] V. E. Gusev, V. Aleshin, and V. Tournat, Acoustic Waves in an Elastic Channel near the Free Surface of Granular Media, Phys. Rev. Lett. 96, 214301 (2006).

[22] S. Brûlé, E. H. Javelaud, S. Enoch, and S. Guenneau, Experiments on Seismic Metamaterials: Molding Surface Waves, Phys. Rev. Lett. 112, 133901 (2014).

[23] S. Krödel, N. Thomé, and C. Daraio, Wide band-gap seismic metastructures, Extrem. Mech. Lett. 4, 111 (2015).

[24] G. Gazetas, Vibrational characteristics of soil deposits with variable wave velocity, Int. J. Numer. Anal. Methods Geomech. 6, 1 (1982).

[25] P. Dakoulas and G. Gazetas, A class of inhomogeneous shear models for seismic response of dams and embankments, Int. J. Soil Dyn. Earthquake Eng. 4, 166 (1985). 